\title{
Temperature anisotropy and differential streaming of solar wind ions. Correlations with transverse fluctuations
}

\author{
S. Bourouaine ${ }^{1}$, E. Marsch ${ }^{1}$, and F. M. Neubauer ${ }^{2}$ \\ ${ }^{1}$ Max-Planck-Institut für Sonnensystemforschung, 37191 Katlenburg-Lindau, Germany \\ e-mail: bourouaine@mps.mpg.de \\ 2 Institut für Geophysik und Meteorologie, Universität zu Köln, Albertus-Magnus-Platz, Köln 50923, Germany
}

Received 10 August 2011 / Accepted 7 October 2011

\begin{abstract}
We study correlations of the temperature ratio (which is an indicator for perpendicular ion heating) and the differential flow of the alpha particles with the power of transverse fluctuations that have wave numbers between 0.01 and 0.1 (normalized to $k_{\mathrm{p}}=1 / l_{\mathrm{p}}$, where $l_{\mathrm{p}}$ is the proton inertial length). We found that both the normalized differential ion speed, $V_{\alpha \mathrm{p}} / V_{\mathrm{A}}$ (where $V_{\mathrm{A}}$ is the Alfvén speed) and the proton temperature anisotropy, $T_{\perp \mathrm{p}} / T_{\| \mathrm{p}}$, increase when the relative wave power is growing. Furthermore, if the normalized differential ion speed stays below 0.5 , the alpha-particle temperature anisotropy, $T_{\perp \alpha} / T_{\| \alpha}$, correlates positively with the relative power of the transverse fluctuations. However, if $V_{\alpha \mathrm{p}} / V_{\mathrm{A}}$ is higher than 0.6 , then the alpha-particle temperature anisotropy tends to become lower and attain even values below unity despite the presence of transverse fluctuations of relatively high amplitudes. Our findings appear to be consistent with the expectations from kinetic theory for the resonant interaction of the ions with Alfvén/ion-cyclotron waves and the resulting wave dissipation.
\end{abstract}

Key words. plasmas - solar wind - turbulence

\section{Introduction}

The in-situ measurements of protons and heavier ions in fast solar wind revealed distinct non-thermal kinetic features, such as the core temperature anisotropy and beam of the protons, or the preferential heating and acceleration of alpha particles and other minor species (see, e.g., the review of Marsch 2006, on this subject). Similarly, solar remote-sensing observations of coronal holes, which are known as the sources of the fast solar wind provided evidence via significant broadenings of ultraviolet emission lines, for strong perpendicular heating of oxygen and heavy ions in the solar corona (see, e.g., the review of Cranmer 2009).

These observed kinetic features have usually been interpreted as signatures of heating by ion-cyclotron waves (Isenberg et al. 2001; Marsch \& Tu 2001; Hollweg \& Isenberg 2002; Matteini et al. 2007). Their origin remains unclear, though, and is still a matter of debate. Some authors suggested that a nonlinear parallel cascade (via parametric decay) of lowfrequency Alfvén waves may ultimately generate ion-cyclotron waves, since their numerical simulations showed that protons and heavy ions can be heated perpendicularly by wave absorption through cyclotron resonance (Araneda et al. 2008, 2009). Moreover, it has been argued theoretically that in low-beta plasma condition, the ion temperature anisotropy and the preferential (stochastic) heating of heavy species could even be caused by Alfvénic fluctuations with frequencies well below the local ion-cyclotron frequency (Wu \& Yoon 2007; Bourouaine et al. 2008; Chandran 2010).

On the other hand, it has also been argued that the energy of Alfvénic turbulence does not cascade effectively to highfrequency parallel cyclotron waves, but rather is transferred into low-frequency and highly oblique kinetic Alfvén waves. Then dissipation would still take place at the proton gyroscale, but via Landau damping acting mostly on electrons (Howes et al. 2008).

In this letter, while benefiting from the high-resolution magnetic field data and the detailed proton and alpha-particle velocity distribution functions (VDFs) obtained (Marsch et al. 1982) simultaneously by the Helios 2 wave and plasma experiments, we analysed typical solar wind data from a heliocentric distance of about $0.7 \mathrm{AU}$. While studying them in detail we found that the prominent kinetic features of the alpha particles and protons are closely correlated with the power of the transverse highfrequency waves. The results of our study place new empirical constraints on solar wind models and further limit the theoretical assumptions involved. We will first present a statistical analysis of the relevant plasma and field parameters, and then discuss them in the light of kinetic plasma theory.

\section{Observations}

Here we analyse plasma and magnetic field data provided by the Helios 2 spacecraft in 1976. For the purpose of a solid statistical study, we selected a week of continuous measurements, i.e., the days with DOY (day of year) numbers 67 to 73 , on which the spacecraft was at a solar distance of about $0.7 \mathrm{AU}$.

As is obvious from Fig. 1, the selected set covers data from measurements made in slow and fast solar wind. The slightly inclined dashed line in that figure separates the data obtained in fast solar wind from those in slow wind. As we would expect, the domain with a relatively high number of collisions mainly corresponds to slow solar wind (with $A_{\mathrm{c}}>0.2$ ), and that with a low number of collisions corresponds to fast solar wind which is weakly collisional (with $A_{\mathrm{c}}<0.1$ ). Here collisionality is quantified by the so-called collision age (see e.g., Salem et al. 2003), 


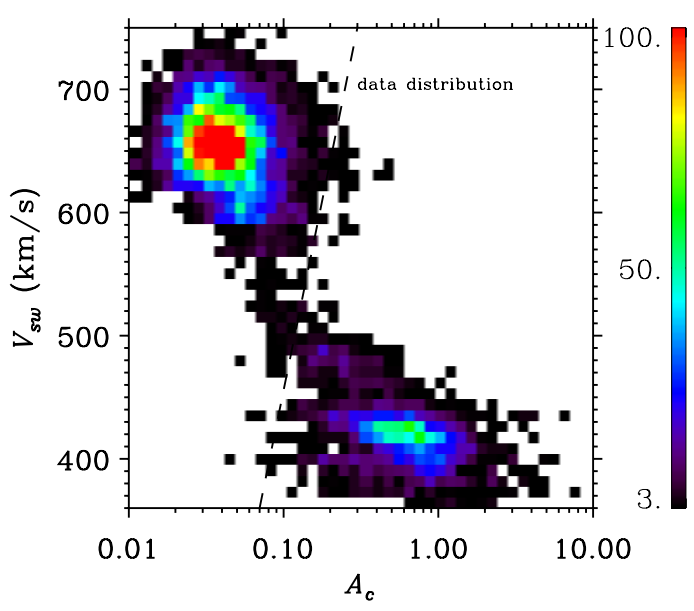

Fig. 1. Distributions of the number of data (presented in colour with the coding indicated by the right-hand bar), which are plotted as a function of the collision age, $A_{\mathrm{c}}$, and the solar wind speed, $V_{\mathrm{sw}}$. The inclined dashed line separates the data from fast and slow solar wind.

$A_{\mathrm{c}}=l /\left(V_{\mathrm{sw}} \tau_{\alpha \mathrm{p}}\right)$, where, $l$ is the distance from the sun and $\tau_{\alpha \mathrm{p}}$ is the collision-exchange time between helium ions and protons. Moreover, for this data set the proton parallel plasma beta, $\beta_{\| \mathrm{p}}$, is comparatively small and mainly varies between 0.1 and 0.7 .

Because the solar wind speed is clearly higher than the Alfvén speed, we can safely assume the so-called Taylor hypothesis to be valid. Therefore, the spacecraft frequency of the magnetic field fluctuations (obtained from fast Fourier transform) is simply given by, $2 \pi f_{\mathrm{sc}} \simeq k V_{\mathrm{sw}}$, where $V_{\mathrm{sw}}$ is the solar wind speed, and $k$ is the wave number of the magnetic field fluctuations. Here, we deal with the average wave power density, $\delta B^{2}$, which is obtained in the spacecraft frame by integration over a frequency range that corresponds to the wave-number range $(0.01-0.1) k_{\mathrm{p}}$ (where $k_{\rho}=1 / l_{\mathrm{p}}, l_{\mathrm{p}}$ is the proton inertial length). Therefore, we are dealing with turbulent fluctuations that still belong to the inertial range but are near the dissipation range of the turbulence. We have chosen this frequency domain to avoid the inclusion of higher-frequency fluctuations that might stem from local wave excitation (e.g., owing to a large temperature anisotropy or high normalized ion differential speed) below but near the proton gyro-kinetic scale. Consistently with our choice of this narrow frequency range, we define the mean field (required as the reference value for the superposed fluctuations) by the average of the full magnetic-field vector over the short time period of about one hundred times $l_{\mathrm{p}} / V_{\mathrm{sw}}$.

In Fig. 2a the distribution of the normalized wave power, $\delta B^{2} / B_{0}^{2}$, is plotted versus the proton temperature anisotropy, $T_{\perp \mathrm{p}} / T_{\| \mathrm{p}}$. It turns out that the latter parameter ranges between 0.4 and 2 and correlates positively with the normalized wave power. According to Fig. $2 b$, the relative compressive component (coded in colour) of the fluctuations is fairly small and not higher than $5 \%$ of the overall wave amplitude, which means that the fluctuations are mainly transverse and essentially incompressible. It also appears that there is no striking correlation between magnetic compressibility and normalized wave power. Unfortunately, we do not have the simultaneous smallscale velocity-fluctuations owing to the lack of an adequate time resolution of the plasma experiment, and therefore we cannot scrutinize the nature of the magnetic fluctuations or confirm by polarization study whether they are Alfvénic or not. But presumably they are Alfvén waves, because these waves with periods
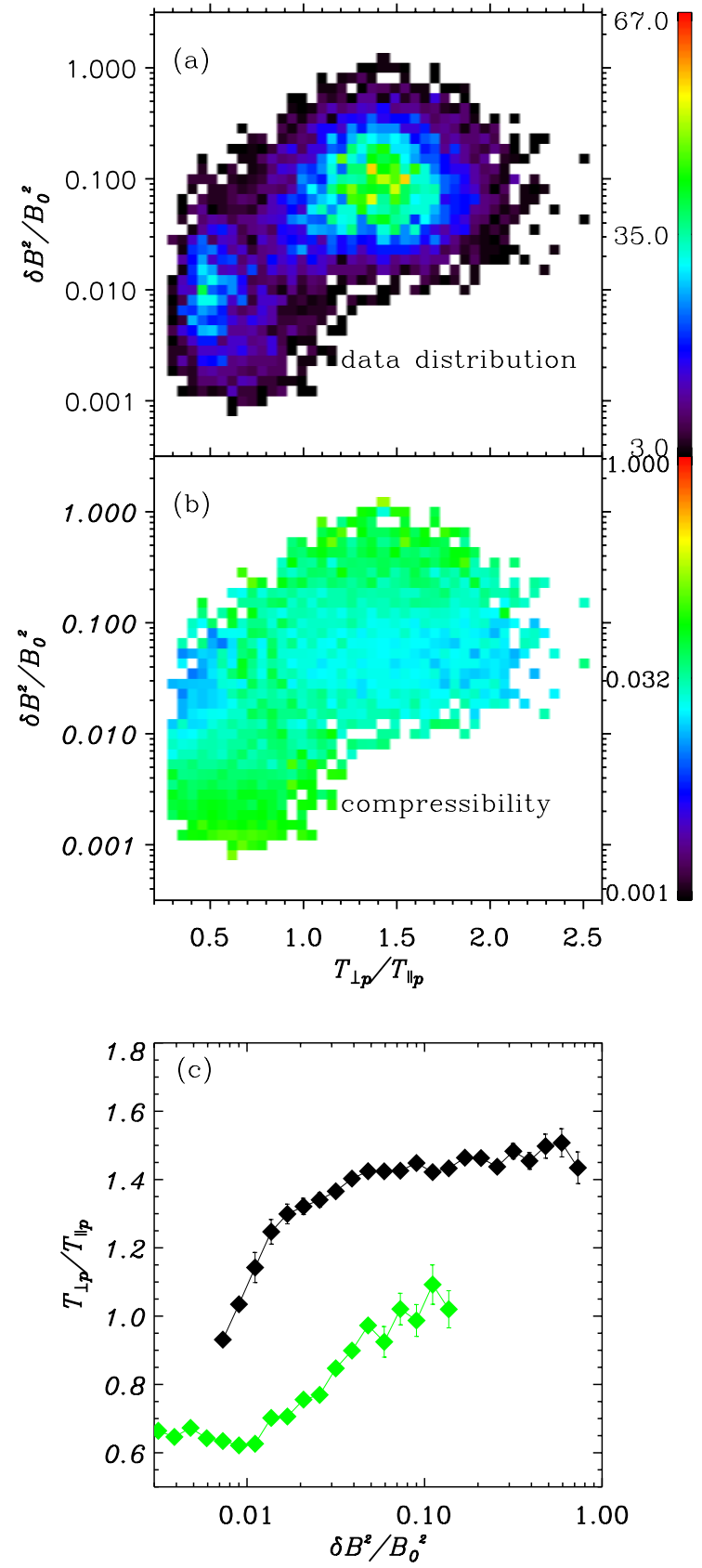

Fig. 2. Distributions (presented in colour with coding indicated by the right-hand bars) of the number of our data a) and the compressibility, $\delta B_{\|}^{2} /\left(\delta B_{\perp}^{2}+\delta B_{\|}^{2}\right)$ b) plotted in the parameter plane of the normalized wave power versus the proton temperature anisotropy, $T_{\perp \mathrm{p}} / T_{\| \mathrm{p}}$; c) weighted mean value of the proton temperature anisotropy for $A_{\mathrm{c}}<$ 0.1 (black), and $A_{\mathrm{c}}>0.2$ (green) displayed versus normalized wave power. The short vertical bars indicate the small uncertainties of the weighted mean values.

longer than $40 \mathrm{~s}$ are observed widely in fast solar wind (Tu \& Marsch 1995) streams at all distances between 0.3 and 1 AU.

In Fig. 2c we plot the weighted mean value (expectation value) of the proton temperature anisotropy as a function of the normalized wave power for different solar wind regimes. In fast solar wind (i.e., where $A_{\mathrm{c}}<0.1$, black curve), the proton temperature anisotropy is below unity whenever the value of the normalized wave power is below 0.01. However, above 0.01 the temperature anisotropy steeply increases until the normalized wave power reaches a value of about 0.1 , where the temperature 
anisotropy tends to saturate at a value of about 1.5 . In contrast, when collisions are relatively strong (i.e., where $A_{\mathrm{c}}>0.2$, green curve), the proton temperature anisotropy also increases with the wave amplitude but reaches lower values than those when collisions are weak. The temperature anisotropy ranges between 0.6 and about 1.1, while the normalized wave power varies between 0.01 and 0.1 .

Our study shows that the proton temperature anisotropy correlates positively with the normalized wave power. Moreover, a positive correlation between the wave power and the proton temperature anisotropy was found earlier by Bourouaine et al. (2010), but for another set of Helios data restricted to fast solar wind and at various solar distances. However, the Helios measurements selected for the present study were made at a fixed heliocentric distance which is about 0.7 AU. Therefore, the new correlation established here between the proton temperature anisotropy and normalized wave power is certainly of local nature and not affected by radial evolution.

According to Fig. 2c, it seems that collisions play a major role in reducing the effects waves have on the local heating of the protons. Therefore, protons can become heated (if wave energy is available) more easily in regions with relatively weak collisions than in regions where collisions are relatively strong.

Figure 3 a shows the data distribution of the normalized wave power versus the normalized ion differential speed. It hardly exceeds unity, a result which is consistent with the prediction of kinetic theory for a linear plasma instability. The theory says that, whenever the ion differential speed exceeds the Alfvén speed, the plasma should become unstable and excite magnetosonic waves (see, e.g., Li \& Habbal 2000). As Bourouaine et al. (2011) found previously, the present study also indicates a positive correlation between the normalized ion differential speed and the normalized wave power (see the white curve, which represents the weighted mean values of the normalized wave power shown in Fig. 3a).

It is commonly believed (see, e.g., the review of Marsch 2006) that low collisional friction would permit a relatively high differential speed to occur between the two main ion species in the solar wind. This notion is confirmed by the results of our Fig. 3b, which shows that higher values of the normalized ion differential speed correspond to lower collision ages. In slow solar wind, when the value of the collision age is higher than 0.2 , the corresponding normalized differential speed is low (i.e., $V_{\alpha \mathrm{p}} / V_{\mathrm{A}}<0.3$ ), but it is higher than 0.3 for comparatively low values of the collision age (as is indicated later in Fig. 3d).

In Fig. $3 \mathrm{c}$ the coloured pixels represent the ratio of the alphaparticle-to-proton temperature anisotropy, $\left(T_{\perp \alpha} T_{\| \mathrm{p}}\right) /\left(T_{\perp \mathrm{p}} T_{\| \alpha}\right)$, plotted as a function of the relative ion differential speed and the normalized wave power. Interestingly, this figure clearly shows that when $V_{\alpha \mathrm{p}} / V_{\mathrm{A}} \leq 0.4$, the temperature anisotropy of the alpha particles, $T_{\perp \alpha} / T_{\| \alpha}$, is higher than the anisotropy of the protons, $T_{\perp \mathrm{p}} / T_{\| \mathrm{p}}$. However, the ratio of the ion temperature anisotropies tends to decrease to lower values of about 0.6 when $V_{\alpha \mathrm{p}} / V_{\mathrm{A}}>0.6$, as is quantitatively shown in Fig. $3 \mathrm{~d}$.

The curve in Fig. 3d, which represents the weighted mean value of the ratio of the ion temperature anisotropies (black symbols), clearly indicates that alpha particles are preferentially heated (perpendicularly to the mean magnetic field) with respect to the protons whenever $V_{\alpha \mathrm{p}} / V_{\mathrm{A}} \leq 0.4$, and this is true even for a relatively low wave energy (indicated by the white curve in Fig. 3a) and at high collision rates (green symbols).

One would expect that the plasma tends to thermal equilibrium, in coincidence with the lowest values of the normalized ion differential flow speed for a relatively high collision rate.
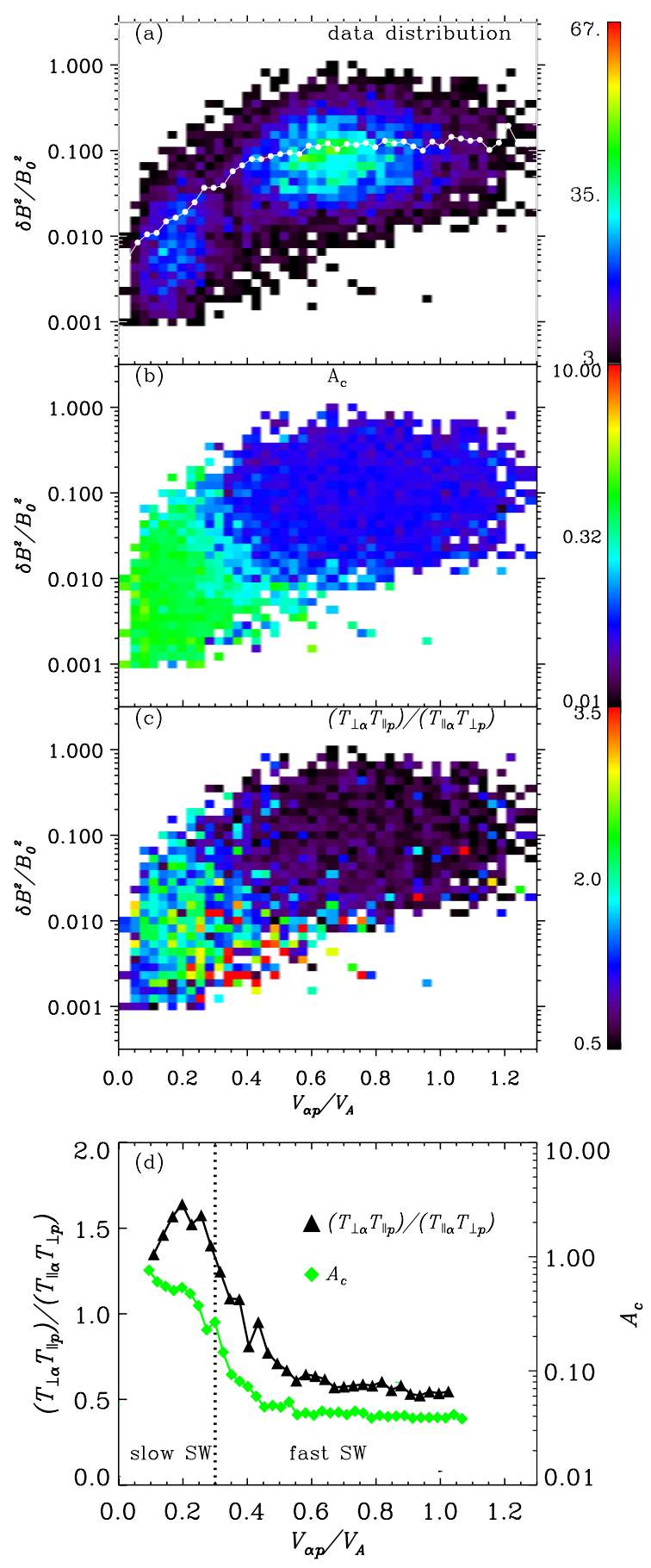

Fig. 3. Distributions (presented in colour with coding indicated by the right-hand bars) of the number of our data a), the collision age, $\left.A_{\mathrm{c}} \mathbf{b}\right)$ and the ratio between the temperature anisotropies of alpha particles and protons, $\left.\left(T_{\perp \alpha} T_{\| \mathrm{p}}\right) /\left(T_{\| \alpha} T_{\perp \mathrm{p}}\right), \mathrm{c}\right)$. All parameter are plotted in the plane of the normalized wave-power, $\delta B^{2} / B_{0}^{2}$, versus normalized ion differential speed, $\left.V_{\alpha \mathrm{p}} / V_{\mathrm{A}} ; \mathbf{d}\right)$ weighted mean values of the collisional age, $A_{\mathrm{c}}$, and the ratio between the temperature anisotropies of alpha particles and protons, $\left(T_{\perp \alpha} T_{\| \mathrm{p}}\right) /\left(T_{\| \alpha} T_{\perp \mathrm{p}}\right)$, displayed versus normalized ion differential speed, $V_{\alpha \mathrm{p}} / V_{\mathrm{A}}$. The white dots in panel a) represent the weighted mean values. The vertical dotted line in panel d) separates the regimes of fast and slow solar wind.

Then the temperature ratios of the ion species should also be near unity. However, observationally it seems that preferential perpendicular heating of the alpha particles with respect to the protons can persist even in regions where collision rates are high 


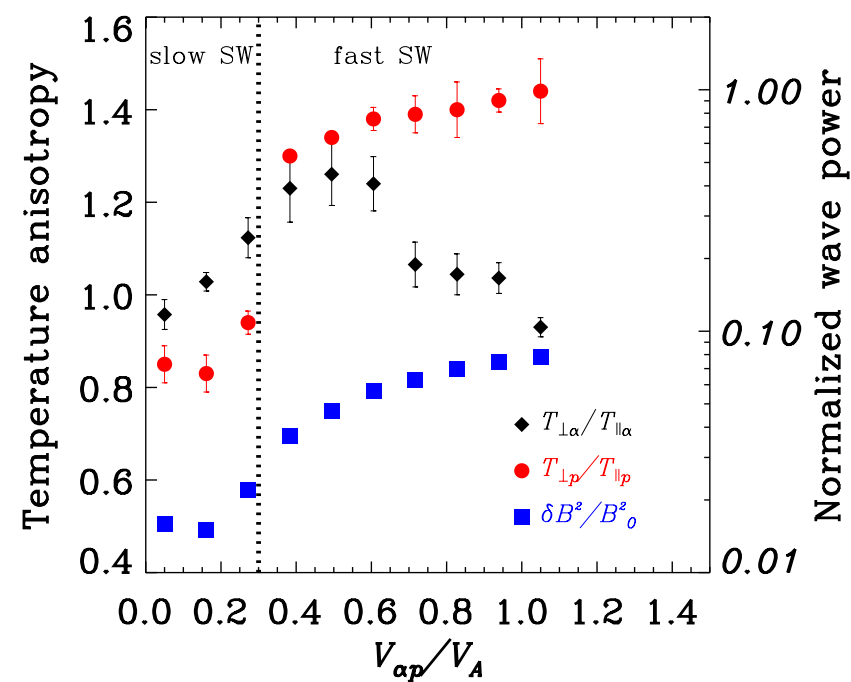

Fig. 4. Left ordinate: the mean values of the temperature anisotropy of the protons, $T_{\perp \mathrm{p}} / T_{\| \mathrm{p}}$ (red dots), and of the alpha particles, $T_{\perp \alpha} / T_{\| \alpha}$ (black diamonds). Right ordinate: mean normalized wave power (blue squares). The bars indicate the uncertainties of the mean values. All quantities are plotted in bins versus the relative ion differential speed, $V_{\alpha \mathrm{p}} / V_{\mathrm{A}}$. The vertical dotted line separates fast from slow solar wind regimes.

(with $V_{\alpha \mathrm{p}} / V_{\mathrm{A}} \leq 0.4$ ). This is possible because a wave-related local ion heating mechanism may be acting on time scales much lower than the long cumulative collision time. Such a fast waveheating mechanism can drive the plasma far away from thermal equilibrium, and therefore may cause a significant ion temperature anisotropy, because on the other side collisions are not fast enough to enforce thermal equilibrium.

We found in our previous paper (Bourouaine et al. 2011) that the helium ion abundance for this selected data set varies mainly between 0.02 and 0.05 . The helium abundance does not show a clear dependence on the normalized differential ion speed. However, we showed that there is an anti-correlation between the alpha-to-proton temperature ratio and the helium abundance at a fixed $V_{\alpha \mathrm{p}} / V_{\mathrm{A}}$.

Figure 4 is a plot of the mean values of the alphaparticle temperature anisotropy, $T_{\perp \alpha} / T_{\| \alpha}$, the proton temperature anisotropy, $T_{\perp \mathrm{p}} / T_{\| \mathrm{p}}$, and the mean relative wave power versus $V_{\alpha \mathrm{p}} / V_{\mathrm{A}}$. The mean values are obtained by averaging the data within bins of a width $\Delta\left(V_{\alpha \mathrm{p}} / V_{\mathrm{A}}\right)=0.1$, and the vertical error bars indicate the related uncertainties of the mean values.

As mentioned above, it turns out that the proton temperature ratio is strictly correlated with the normalized wave power, which indicates perpendicular heating of the protons whenever the power of the transverse fluctuations is enhanced.

An interesting behaviour of the temperature ratio of the alpha particles can be inferred from Fig. 4. This ratio increases with increasing normalized wave power as long as the normalized ion differential speed stays below about 0.5 . Beyond this value of $V_{\alpha \mathrm{p}} / V_{\mathrm{A}}$, the alpha-particle temperature ratio becomes roughly constant, until $V_{\alpha \mathrm{p}} / V_{\mathrm{A}}$ exceeds a value of about 0.7 , but then it decreases towards a value below unity when $V_{\alpha \mathrm{p}} / V_{\mathrm{A}}$ reaches one. In the slow solar wind region, where the collisions are expected to be relatively high, the proton temperature anisotropy is ranging between 0.8 and 1 , however, the alpha temperature anisotropy is not in the same range but higher, and varies between 0.9 and 1.2 .
Most likely strong collisionality at low $V_{\alpha \mathrm{p}} / V_{\mathrm{A}}$ tends to isotropize the alphas, whereas the weakening of the resonance at higher alpha/proton speeds leads to a decreasing of the alpha anisotropy, as in Gary et al. (2005).

The monotonic increase of the proton temperature anisotropy with the normalized differential ion speed appears to be consistent with the findings of Kasper et al. (2008) and with the ACE observations of Gary et al. (2005). However, there is clear difference between the trend of the alpha temperature anisotropy in Fig. 4 and the results found by Kasper et al. (2008). In their paper, the alpha temperature anisotropy reaches a minimum value at $V_{\alpha \mathrm{p}} \sim 0.5 V_{\mathrm{A}}$, whereas our Fig. 3 shows that the alpha temperature anisotropy reaches its maximum value when $V_{\alpha \mathrm{p}} \sim 0.5 V_{\mathrm{A}}$. Moreover, the results of Gary et al. (2005) show that the average alpha temperature anisotropy is monotonically decreasing with increasing alpha/proton relative speed.

Unlike what Kasper et al. (2008) and Gary et al. (2005) found previously, our Fig. 4 shows that the perpendicular heating is reduced when $V_{\alpha \mathrm{p}} / V_{\mathrm{A}}$ is near zero. This reduced ion heating corresponds to an observed concurrent decrease in the wave power of the transverse waves. This results would be expected when the heating ultimately rests with the energy in those waves. If $V_{\alpha \mathrm{p}} / V_{\mathrm{A}}$ is near zero, we expect that the alpha-particle temperature ratio increases resulting in a strong perpendicular ion heating for sufficient wave power. However, although the wave power is empirically found to be weak when the normalized ion differential speed is low, the alpha particles are still heated perpendicularly much more than the protons. For both ion species the interaction with transverse waves is expected to work against the radial trend caused by the solar wind expansion in a magnetic mirror, which tends to build up a much larger parallel than perpendicular temperature, and accordingly a strong fire-hose-type anisotropy.

\section{Discussion and conclusions}

If we assume that the transverse fluctuations provide the energy input for the observed preferential perpendicular heating of the alpha particles, then their temperature-ratio profile as given in Fig. 4 could be a signature of cyclotron-wave heating of the alpha particles.

It has been claimed that the alpha particles can only be heated through ion-cyclotron wave dissipation if the differential speed between alpha-particles and protons is approximately less than $0.5 V_{\mathrm{A}}$ (see e.g., Gary et al. 2005). Therefore, alpha-particles can be heated in the perpendicular direction as long as they stay in resonance with the ion-cyclotron waves. Moreover, the wave energy is an important input parameter that controls the heating of alpha-particles. We expect that high wave energy can cause strong perpendicular heating of those alpha particles that are in resonance with ion-cyclotron waves.

Figure 4 shows that the perpendicular heating is reduced when $V_{\alpha \mathrm{p}} / V_{\mathrm{A}}$ is near zero. This reduced ion heating corresponds to a decrease in the observed wave power of the transverse fluctuations, a result that is expected because the potential for heating ultimately rests with the energy contained in those waves. If $V_{\alpha \mathrm{p}} / V_{\mathrm{A}}$ is near zero, we expect that the alpha-particle temperature ratio increases, resulting in strong perpendicular ion heating for sufficient wave power. However, although the wave power is found empirically to be weak when the normalized ion differential speed is small, the alpha particles can still be perpendicularly heated much more than the protons. Yet, for both ion species the interaction with transverse fluctuations works against the trend 
caused by the solar wind expansion in a magnetic-field mirror configuration, which tends to build up a high parallel temperature anisotropy. Furthermore, Coulomb collisions tend to thermalize the solar wind plasma and to reduce the differential speed between alpha particles and protons. But collisions are effective in removing the non-thermal ion features merely in the comparatively cold and dense slow solar wind, in which the collision age is found to be high and the average wave power observed to be weak.

Another possible scenario that may occur as well is that the long-wavelength and high-amplitude fluctuations of the inertialrange turbulence may stochastically heat (Chandran 2010) the ions in the perpendicular direction with respect to the background magnetic field (or non-resonantly drive a slowly varying $T_{\perp} / T_{\|}$on both ion species). The presence of this anisotropy can give rise to Alfvén-cyclotron instabilities, which lead to the growth of relatively high-frequency modes (with frequencies $\omega \sim \Omega_{\mathrm{p}}$, where $\Omega_{\mathrm{p}}$ is the proton cyclotron frequency). In other words, the ion temperature anisotropies could first be caused by low-frequency fluctuations in the inertial range, and then this thermal energy may be exchanged between ions and waves at the proton kinetic scale.

\section{References}

Araneda, J. A., Marsch, E., \& Viñas, A. F. 2008, Phys. Rev. Lett., 100, 125003 Araneda, J. A., Maneva, Y., \& Marsch, E. 2009, Phys. Rev. Lett., 102, 175001 Bourouaine, S., Marsch, E., \& Vocks, C. 2008, ApJ, 684, L119

Bourouaine, S., Marsch, E., \& Neubauer, F. M. 2010, Geophys. Res. Lett., 37, L14104

Bourouaine, S., Marsch, E., \& Neubauer, F. M. 2011, ApJ, 728, L3

Chandran, B. D. G. 2010, ApJ, 720, 548

Cranmer, S. R. 2009, Liv. Rev. Sol. Phys., 6, 3

Gary, P. S., Smith, C. W., \& Skoug, R. M. 2005, J. Geophys. Res., 110, 108

Howes, G. G., Dorland, W., Cowley, S. C., et al. 2008, Phys. Rev. Lett., 100, 065004

Matteini, L., Landi, S., \& Hellinger, P. 2007, Geophys. Res. Lett., 34, L20105

Hollweg, J. V., \& Isenberg, P. A. 2002, J. Geophys. Res., 107, 1147

Isenberg, P. A., Lee, M. A., \& Hollweg, J. V. 2001, J. Geophys. Res., 106, 5649

Kasper, J. C., Lazarus, A. J., \& Gary, S. P. 2008, Phys. Rev. Lett., 101, 261103

Li, X., \& Habbal, S. R. 2000, Geophys. Res., 105, 7483

Marsch, E. 2006, Liv. Rev. Sol. Phys., 3, 1

Marsch, E., \& Tu, C.-Y. 2001, J. Geophys. Res., 106, 227

Marsch, E., Schwenn, R., Rosenbauer, H., et al. 1982, J. Geophys. Res., 87, 52

Salem, C., Hubert, D., Lacombe, C., et al. 2003, ApJ, 585, 1147

Tu, C.-Y., \& Marsch, E. 1995, Space Sci. Rev., 73, 1

Wu, C. S., \& Yoon, P. H. 2007, Phys. Rev. Lett., 99, 075001 